\title{
Nuclear Weak Processes in Presupernova Stars
}

\author{
By A. RA Y, ${ }^{1}$ T. $\mathbf{K A ~ R ,}{ }^{2}$ \\ S. S A R K A R ${ }^{2}$, AND S. CHAKRAVART $\mathbf{I}^{3}$ \\ ${ }^{1}$ Tata Institute of Fundamental Research, Bombay 400 005, India \\ ${ }^{2}$ Saha Institute of Nuclear Physics, Calcutta 700 064, India \\ ${ }^{3}$ California State Polytechnic University, Pomona, CA91768, USA
}

The structure and the size of the core of massive presupernova stars are determined by the electron fraction and entropy of the core during its late stages of evolution; these in turn affect the subsequent evolution during gravitational collapse and supernova explosion phases. Beta decay and electron capture on a number of neutron rich nuclei can contribute substantially towards the reduction of the entropy and possibly the electron fraction in the core. Methods for calculating the weak transition rates for a number of nuclei for which no reliable rates exist (particularly for $A>60$ ) are outlined. The calculations are particularly suited for presupernova matter density $\left(\rho=10^{7}-10^{9} \mathrm{~g} / \mathrm{cc}\right)$ and temperature $\left(\mathrm{T}=2-6 \times 10^{9}{ }^{\circ} \mathrm{K}\right)$. We include besides the contributions from the ground state and the known excited states, the GamowTeller $(G T)$ resonance states (e.g. for beta decay rates, the $G T_{+}$states) in the mother nucleus which are populated thermally. For the $G T$ strength function for transitions from the ground state (as well as excited states) we use a sum rule calculated by the spectral distribution method where the centroid of the distribution is obtained from experimental data on $(p, n)$ reactions. The contribution of the excited levels and $G T_{+}$resonances turn out to be important at high temperatures which may prevail in presupernova stellar cores.

\section{Presupernova Evolution of Massive Stars}

Beta decay $\left(\beta^{-}\right)$and electron $\left(\mathrm{e}^{-}\right)$capture of neutron rich nuclei play important roles in determining presupernova core structure (Nomoto et al, 1991). The structure of the core is important for later gravitational collapse and supernova explosion phases. These weak interaction rates were compiled by Fuller et al. (1982 and references therein) for a series of nuclei up to the mass number (A) of 60 . Reliable rates for $A>60$ are not available yet. It is important to include contributions of excited states of parent nuclei because presupernova cores are hot and dense. In addition there are resonance states in the mother nucleus whose contribution to the overall rates in a hot presupernova core have to be accounted for. For considerations of beta-decay rates for example, the Gamow-Teller $_{+}$resonances may be thermally populated and the transitions from these states could in principle be important. The distribution of the $G T_{+}$strengths have been recently obtained experimentally in a few nuclei from $(n, p)$ reactions (e.g. Alford et al. 1993) and can be used in calculating the transition strengths from the resonance states.

In the core of the star, the last stage of nuclear fusion involves silicon. When silicon burning is finished the core tends to contract under its own weight. During the contraction phase (the so-called Kelvin-Helmholtz phase) the iron group nuclei capture electrons and emit neutrinos which escape directly from the stellar core without further interaction. This leads not only to a decreased electron fraction, $\mathrm{Y}_{e}$, but also to lower entropy per nucleon and a cooler core. In addition, the entropy profile has significant effects on presupernova core structure and nucleosynthesis, since the former determines the extent of the convective burning shells. Woosley \& Weaver (1988) have emphasized 
that electron captures can lead to some neutronization during oxygen burning and in particular after central oxygen depletion. Even though the final distribution of $Y_{e}$ in the $\mathrm{Si}$ core will have little memory of what the conditions during oxygen burning were, a decrease of $\mathrm{Y}_{e}$ during oxygen burning might affect the entropy and density structure of the outer core and through this, the location of the oxygen burning shell during the core silicon burning stage. Because of its entropy generation, the location of the oxygen burning shell does ultimately determine the extent of the convective Si burning shell that follows and sets the iron core mass.

So far, out of the weak processes, $\beta^{-}$decays of nuclei are not yet included in the computation of presupernova stellar structure. The electron capture processes are included in the computations (Woosley \& Weaver 1988) by using the instantaneous value of the neutron excess (or $\mathrm{Y}_{e}$ ) at time $\mathrm{t}$. The abundances of radioactive nuclei (computed from $\rho, \mathrm{T}$, and $\mathrm{Y}_{e}$ ) and their decay rates $\lambda_{i}$ yield the rate at which $\mathrm{Y}_{e}$ is changing. Obviously in computing the change of $Y_{e}$, one must know whether the nuclei with the largest rates are abundant enough under the given conditions of density, temperature and electron fraction. In general, such abundances can be computed only through detailed reaction networks coupled dynamically to stellar evolution. Hence it is necessary to compute capture and decay rates of nuclei under conditions prevailing in the presupernova stage, especially for nuclei in the fp-shell.

For the fp-shell, detailed shell model calculations to obtain rates are formidable computationally. Bloom \& Fuller (1985) tried such methods for nuclei with $A<60$ using a truncated basis space. Among microscopic theories, the best results are from complete shell model diagonalisation for $\mathrm{A}>40$ nuclei (Brown \& Wildenthal 1985) and making a Quasiparticle Random Phase Approximation (QRPA) for heavier nuclei (Moller \& Randrup, 1990; Klapdor et al. 1990). However, extension of the QRPA calculations to non-zero temperatures relevant for presupernova and gravitational collapse phases leading to supernovae are not available yet.

\section{Calculation of Rates}

Following Kar et al. (1991), the we use Gamow-Teller sum rules and employ the average distribution of the quenched sum-rule strength calculated from spectral distribution theory (see French et al. 1988; Kota \& Kar 1989). A continuous approximation is made for the final states. We calculate the variable phase space factors (as a function of final energy) for the reaction rates using the finite temperature Fermi-Dirac distributions. A filled Fermi-sea of electrons partially blocks the available phase space. We also numerically calculate the electron chemical potential (required in phase space integrals) in the moderately degenerate and mildly relativistic cases. The $G T$ strength distribution is folded in with the variable phase space factor and integrated over the final state energy to yield the rate from a particular mother nucleus state. This procedure is repeated to include the statistically weighted contributions from excited states of the mother nucleus for finite temperature.

The $\beta^{-}$decay rate from the ground state is given by:

$$
\lambda_{g . s}=\frac{\ln 2}{\left(g_{V}\right)^{2}}(6250 \mathrm{~s})^{-1} \sum_{j} B\left(E_{j}\right) f\left(Q-E_{j}\right)
$$

where the summation is over all final states with energy $E_{j}$ up to the Q-value.

$$
B\left(E_{j}\right)=g_{V}^{2} B_{F}\left(E_{j}\right)+g_{A}^{2} B_{G T}\left(E_{j}\right),
$$

$\left[B_{F}(E)\right.$ and $B_{G T}(E)$ are the squared Fermi and $G T$ matrix elements]. We replace the 
actual microscopic strengths by the statistically averaged strength function and approximate the $\sum_{j} \rightarrow \int d E^{\prime}$ over $Q_{i}=Q+E_{i}$ in the rate equation for $\lambda_{g s}$. Therefore the decay rate from the ground state as well as the known excited states taking account of the Boltzmann factors is:

$$
\lambda_{s}=\frac{\ln 2}{\left(g_{V}\right)^{2}} \frac{(6250 \mathrm{~s})^{-1}}{G} \sum_{i}\left(2 J_{i}+1\right) \exp \left(-E_{i} / k T\right) \int_{0}^{Q_{i}}\left|M_{i}\left(E^{\prime}\right)\right|^{2} f\left(Q_{i}-E^{\prime}\right) d E^{\prime}
$$

with

$$
\left|M_{i}\left(E^{\prime}\right)\right|^{2}=g_{V}^{2} \rho_{i}\left(E^{\prime}\right) B_{i}^{F}\left(E^{\prime}\right)+g_{A}^{2} \rho_{i}\left(E^{\prime}\right) B_{i}^{G T}\left(E^{\prime}\right) .
$$

Here $\rho_{i}\left(E^{\prime}\right)$ is the density of states at final energy $E^{\prime}$. The phase-space factor appearing in the equation for the transition rate is:

$$
f\left(T, \mu, E_{0}\right)=\int_{1}^{\epsilon_{0}} \frac{F(Z, \epsilon) \epsilon\left(\epsilon^{2}-1\right)^{1 / 2}\left(\epsilon_{0}-\epsilon\right)^{2} d \epsilon}{1+\exp \{(\mu-\epsilon) / k T\}}
$$

The factor in the denominator denotes the availability of electron holes in the Fermi sea which the decay electron can enter. $F(Z, \epsilon)$ is the Coulomb correction factor (Schenter $\&$ Vogel 1983) and incorporates the effect of relativistic electrons in the presupernova interior and corrects for the finite size of the nucleus. The actual integrals for the phasespace factor and transition rate are obtained by 16 point Gauss-Legendre quadratures. The central temperature at the last nuclear burning stages approach a good fraction of an $\mathrm{MeV}$. Therefore the chemical potential of the electrons are calculated fully numerically at finite temperatures. This includes the effects of the presence of positrons (through electron-positron pair creation).

Apart from the contribution of the ground and the known excited states of the nucleus, the contribution from the $G T_{+}$resonance in the mother nucleus for $\beta^{-}$decay $\lambda_{R E}^{>}$must be added to get the total rate:

$$
\lambda_{t o t}=\lambda_{s}+\lambda_{R E}^{>}
$$

\section{GT Strength Sum and Energy Distribution}

For the position of the IAS, the Coulomb displacement energy is: $\Delta_{c}=1.44 Z A^{-1 / 3}$ $\mathrm{MeV}$ and the width is $\sigma_{c}=0.157 Z A^{-1 / 3} \mathrm{MeV}$ (Morita 1973). For the fp-shell nuclei, as the very narrow Fermi resonance lies far above the energetically allowed region, the Fermi operator makes a negligible contribution to $\beta^{-}$decay rates. However, the collective Gamow-Teller mode can be substantially split among the daughter nuclear levels as compared to the Fermi IAS. See Figure 1. We use the spectral distribution theory for evaluating Gamow-Teller strength sum and the form of the strength distribution. The statistically averaged smoothed form of the Gamow-Teller strength function is a Gaussian in energy $E^{\prime}$ because according to spectral distribution theory the strength density (i.e. the average strength times the density of states in both the initial and final spaces) has an asymptotic bivariate Gaussian form in the initial and final energies for large spaces (French et al. 1988). Experimentally observed total $G T$ strengths from the ground state of the target nucleus through $(p, n)$ reactions show that the rates are consistently smaller than estimates from the shell model or other theoretical models. Movement of a part of the total strength upward in energy beyond the observed giant resonance region due to the tensor interaction as well as due to mixing of $N^{-1} \Delta$ (nucleon-hole $\Delta$ particle) with the low-energy $N^{-1} N$ interaction is believed to be the cause of this observed quenching. We take this into account by an including an empirical quenching factor

To specify the Gaussian one needs two quantities - its centroid and its width. We 


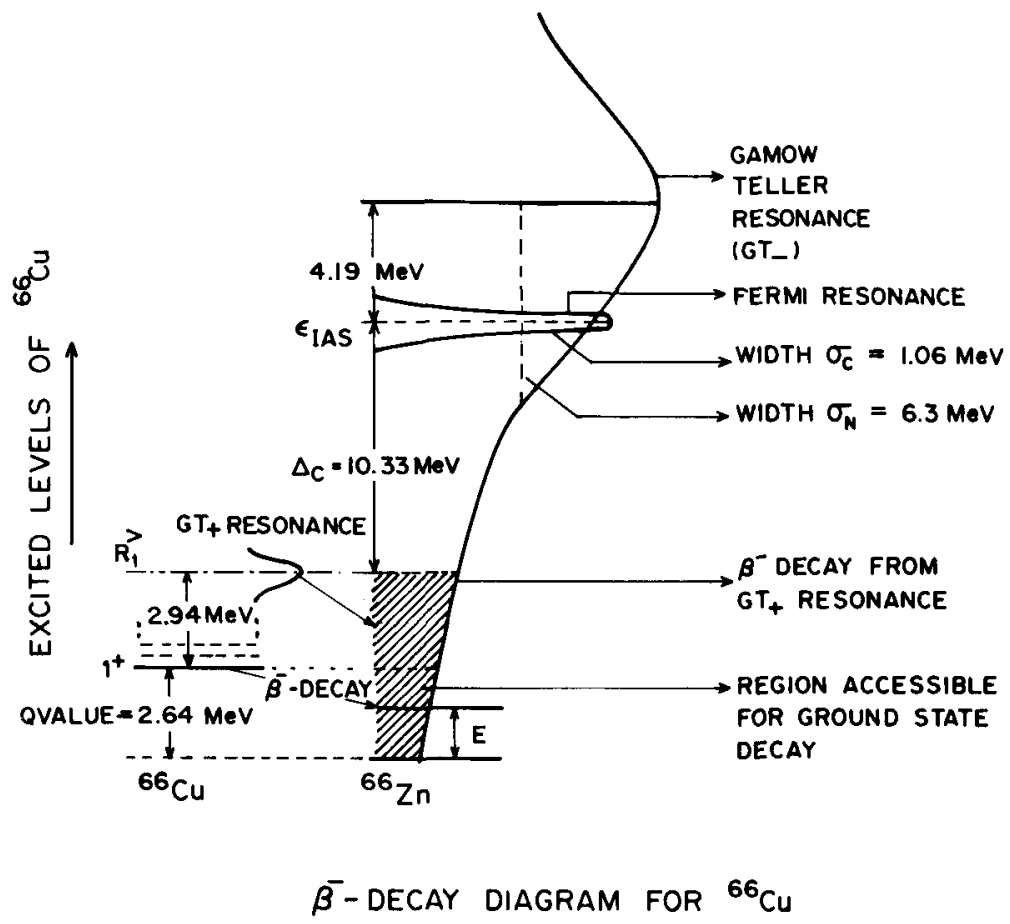

Figure 1. $\beta^{-}$decay diagram for ${ }^{66} \mathrm{Cu}$ with Fermi and $G T$ resonances

note here that the physically accessible region through the Q-value of the ground state (the excited states) covers only the tail of the Gaussian. For the GT centroid we use the relation developed by Nakayama et al. (1982) which gives a best-fit to the observed centroids from $(\mathrm{p}, \mathrm{n})$ reaction data. The expression is

$$
\epsilon_{G T}=E_{I A S}+26 A^{-1 / 3}-18.5(N-Z) / A
$$

where $\epsilon_{G T}$ is the $G T$ centroid energy and $E_{I A S}$ is the energy of the IAS. For an excited state, we extend the isobaric analog argument and add the excitation energy of the initial state to fix the centroid energy of its $G T$ distribution. The width of the $G T$ resonance has two parts: $\sigma^{2}=\sigma_{C}^{2}+\sigma_{N}^{2}$, the first part coming from Coulomb forces and the dominant second part coming from the nuclear interactions. To minimize the deviation of the calculated strength distribution from the actual one, we use the width of the Gaussian as a parameter and fit the experimental half-lives with predictions of the model for a number of nuclei.

\section{Transitions from Gamow-Teller Resonances in the Mother Nucleus}

For $\beta^{-}$decay, apart from the transitions from individual excited states and the ground state of the mother nucleus, one should also include the resonant states of the mother nucleus $(\mathrm{N}, \mathrm{Z})$ which resonates with low-lying states of the daughter nucleus $(\mathrm{N}-1, \mathrm{Z}+1)$ being connected by the $G T_{+}$operator for $\beta^{+}$decay (i.e. $\Sigma_{i} \sigma(i) t_{+}(i)$ ) on the daughter states. (For electron capture reactions, one should include the corresponding $G T_{-}$states 
in the mother nucleus.) Adopting the method of Fuller, Fowler \& Newman (FFN II) where one assumes that the centroid energies of the resonances in the mother scale in the same way as the discrete states in the daughter, a simple form for the transition rates $\left(\lambda_{R E}^{>}\right)$of $\beta^{-}$decay is given by:

$$
\lambda_{R E}^{>}=\lambda_{\text {re }}^{>}\left(\frac{G^{<}}{G^{>}}\right) \exp \left(-R_{1}^{>} / k T\right)
$$

where $G^{<}$and $G^{>}$are partition functions of the daughter and the mother nucleus

$$
G(Z, A, T)=\iint d J d E(2 J+1) \rho(E, J) \exp (-E / k T)
$$

where $\rho(E, J)$ is the density of nuclear levels.

For nuclei whose excitation spectra (of both mother and daughter) are known experimentally, the partition functions are calculated up to the same excitation energy in both. For cases in which such spectra are not known, we use the "constant temperature" form of the nuclear level density functions in the partition function integrals (see e.g. von Egidy et al. 1988):

$$
\rho(E, J)=f(J) \frac{1}{T_{n}} \exp \left(\frac{E-E_{0}}{T_{n}}\right),
$$

where $f(J)$ is the spin distribution function. The back-shifting parameter $E_{0}$ and the nuclear level density function's parameter $T_{n}$ (the "constant temperature") have been given by von Egidy et al. in parametrized fits depending on the nuclear mass number A and on the pairing energies of the protons and neutrons (Gilbert \& Cameron 1965).

In eq. (8), the excitation energy $R_{1}^{>}$of the first resonance state has three parts:

$$
R_{1}^{>}=\Delta E_{s . p}+\Delta E_{p-h}+\Delta E_{\text {pairing }}
$$

where $\Delta E_{s . p}$. is due to single particle excitation energy difference between a lower and a higher orbit, $\Delta E_{p-h}$ is the effect of the two-body residual interaction on the excitation of the particle and $\Delta E_{\text {pairing }}$ is the change in the pairing energy caused by breaking or creation of each pair. For the single particle energies for the neutrons as well as protons we use the sequence and the values as given for neutrons by Hillman \& Grover (1969). For ${ }^{66} \mathrm{Cu}$, the centroid of the first resonance $G T_{+}$is at $2.94 \mathrm{MeV}$ above the ground state of the mother. The strength $\lambda_{r e}^{>}$for each single particle transition (from orbit $r \rightarrow s$ ) is given by

$$
\lambda_{r e}^{>}=\frac{\ln 2}{\left(g_{V}\right)^{2}}(6250 s e c)^{-1}\left|M_{G T}^{s . p}\right|^{2} \frac{n_{p}^{r} n_{h}^{s}}{\left(2 j_{s}+1\right)} f\left(T, \mu, Q+R_{1}^{>}\right)
$$

where $M_{G T}^{s . p}$ is the single particle $G T$ matrix element, $n_{p}^{p}$ is the number of proton particles in orbit $p$ and $n_{h}^{s}$ the number of neutron holes in orbit $s$ (with the total number of particles $\left(2 j_{s}+1\right)$ in the daughter nucleus for its $\beta^{+}$transition). For more than one possible single particle transitions, the strengths have been simply added to get the total $\lambda_{r e}^{>}$to a single state of the daughter nucleus and the energy of that state has been fixed by the scaling argument of FFNII to get the Q-value of the resonance transition. Here, a typical quenching factor of 0.5 is introduced for the $G T_{+}$transition.

\section{Rates for Nuclei in the fp-Shell}

To test the predictions of the model and to fix the parameter $\sigma_{N}$, we calculate the half-lives of the following nuclei in the fp shell with $A>60:{ }^{69} \mathrm{Cu},{ }^{68} \mathrm{Cu},{ }^{67} \mathrm{Ni},{ }^{66} \mathrm{Cu}$, ${ }^{65} \mathrm{Ni},{ }^{65} \mathrm{Co},{ }^{64} \mathrm{Co},{ }^{63} \mathrm{Co},{ }^{63} \mathrm{Fe},{ }^{62} \mathrm{Co},{ }^{62} \mathrm{Fe},{ }^{62} \mathrm{Mn}$ and ${ }^{61} \mathrm{Fe}$. For the 13 nuclei considered, the best fit to experimental value is obtained with $\sigma_{N}=6.3 \mathrm{MeV}$. Table 1 compares the 
TABLE 1. Comparisons of Calculated and Experimental Half-Lives

\begin{tabular}{|c|c|c|c|c|}
\hline Nucleus & Experiment & $\begin{array}{r}\text { Half-life } \tau_{1 / 2}(\mathrm{sec}) \\
\text { This work }\end{array}$ & Gross theory & QRPA \\
\hline${ }^{69} \mathrm{Cu}$ & 171. & 180.1 & & \\
\hline${ }^{68} \mathrm{Cu}$ & 31.1 & 25.4 & & \\
\hline${ }^{66} \mathrm{Cu}$ & 306. & 305.0 & & \\
\hline${ }^{67} \mathrm{Ni}$ & 21. & 46.9 & 94 & 23. \\
\hline${ }^{65} \mathrm{Ni}$ & 9072 . & 589. & & \\
\hline${ }^{62} \mathrm{Co}$ & 90. & 15.1 & & \\
\hline${ }^{63} \mathrm{Co}$ & 27.4 & 52.1 & & \\
\hline${ }^{64} \mathrm{Co}$ & 0.3 & 3.53 & & 10.0 \\
\hline${ }^{65} \mathrm{Co}$ & 1.25 & 5.64 & 8 & 8.59 \\
\hline${ }^{61} \mathrm{Fe}$ & 358.8 & 34.5 & & \\
\hline${ }^{62} \mathrm{Fe}$ & 68. & 197.3 & & \\
\hline${ }^{63} \mathrm{Fe}$ & 6.1 & 3.49 & 10 & 14.8 \\
\hline${ }^{62} \mathrm{Mn}$ & 0.88 & 0.80 & 2 & 0.773 \\
\hline
\end{tabular}

calculated free decay half-lives with the experimental values. It also gives the predictions of microscopic QRPA calculations of Klapdor et al. (1990) as well as those of the gross theory (Takahashi et al. 1973). For ${ }^{64} \mathrm{Co}$, an Edgeworth expansion of the $G T$ strength distribution with negative skewness of -0.3 and a $\sigma_{N}=7.5$ gives the predicted half life to be $0.907 \mathrm{~s}$ (whereas the experimental value is $0.3 \mathrm{sec}$; note the different calculated value reported in Table 1 which is for standard $\sigma_{N}$ and symmetric Gaussian).

The finite temperature and density results for all the nuclei show the general trend that the rates decrease with increasing density of matter in the stellar core. This trend is due to the increasing chemical potential of electrons outside the nuclei impeding the decay process. The phase space factor $f$ is very strongly dependent on the $Q$ value - for free decays $f \propto Q^{5}$ if the Coulomb correction factor $\mathrm{F}(\mathrm{Z}, \mathrm{E})=1$. For transitions from a given state of the mother nucleus to daughter states of increasing excitation, the $Q$-value decreases and so does the phase space factor. On the other hand, the $G T$ strength rises because one goes higher up in energy towards the centroid from the tail region. Thus there is a competition between two opposing effects. The inclusion of excited states can increase the rate by a factor as large as 11.4 as seen for ${ }^{69} \mathrm{Cu}$ at the high density of $\log \rho_{10}=-0.5, T=6 \times 10^{9} \mathrm{~K}$ and $Y_{e}=0.50$.

Table 2 compares the $\beta^{-}$decay rates from the ground state and the known excited states up to $4.85 \mathrm{MeV}$ to the total transition rates from these as well as the $G T_{+}$resonance states. It is clear that the $G T_{+}$resonance state makes an appreciable contribution only at high temperatures. Table 3 gives the electron capture rates of ${ }^{62} \mathrm{Co}$. (The reported rates do not include the contributions from the corresponding $G T_{\text {- resonances in the }}$ mother.) Rates for all 13 nuclei, cannot be reproduced here due to space restrictions but will be published elsewhere.

It is clear from the previous sections that the strength distribution of $G T_{-}$and $G T_{+}$ resonances in daughter and mother nuclei are important in calculations of beta decay rates. These have been estimated on theoretical grounds either through shell-model type calculations, QRPA or spectral distribution theory etc. However, charge exchange reactions (e.g. $(n, p)$ or $(p, n)$ reactions on mother and daughter nuclei for beta decay) 
TABLE 2. $\beta^{-}$decay rates for ${ }^{66} \mathrm{Cu}$ at $\mathrm{Y}_{e}=0.42$

\begin{tabular}{|c|c|c|c|c|}
\hline $\log \rho_{10}$ & $\mathrm{~T}\left({ }^{\circ} \mathrm{K}\right)$ & $\lambda_{g . s .}+\lambda_{e x}$ & $\lambda_{\text {total }}$ & $G^{</ G^{>}}$ \\
\hline-2.0 & $\begin{array}{l}3 \times 10^{9} \\
4 \times 10^{9} \\
5 \times 10^{9}\end{array}$ & $\begin{array}{l}2.65 \times 10^{-3} \\
3.58 \times 10^{-3} \\
4.74 \times 10^{-3}\end{array}$ & $\begin{array}{l}2.65 \times 10^{-3} \\
3.64 \times 10^{-3} \\
5.04 \times 10^{-3}\end{array}$ & $\begin{array}{l}0.076 \\
0.062 \\
0.059\end{array}$ \\
\hline-1.0 & $\begin{array}{l}3 \times 10^{9} \\
4 \times 10^{9} \\
5 \times 10^{9}\end{array}$ & $\begin{array}{l}7.16 \times 10^{-5} \\
2.44 \times 10^{-4} \\
5.87 \times 10^{-4}\end{array}$ & $\begin{array}{l}7.35 \times 10^{-5} \\
2.71 \times 10^{-4} \\
7.32 \times 10^{-4}\end{array}$ & $\begin{array}{l}0.076 \\
0.062 \\
0.059\end{array}$ \\
\hline
\end{tabular}

TABlE 3. Electron Capture Rates for ${ }^{62}$ Co with $Y_{e}=0.50$

\begin{tabular}{ccc}
\hline \hline Temperature $\left({ }^{\circ} \mathrm{K}\right)$ & Capture rates \\
\hline & $\log \rho_{10}=-2.0$ & $\log \rho_{10}=-3.0$ \\
\hline $3 \times 10^{9}$ & $9.25 \times 10^{-7}$ & $1.81 \times 10^{-8}$ \\
$4 \times 10^{9}$ & $6.17 \times 10^{-6}$ & $3.25 \times 10^{-7}$ \\
$5 \times 10^{9}$ & $2.42 \times 10^{-5}$ & $2.31 \times 10^{-6}$ \\
\hline
\end{tabular}

can probe these strength distributions $\left[B\left(G T_{+}\right)\right.$and $\left.B\left(G T_{-}\right)\right]$experimentally and can serve as important input data. Approximately a dozen nuclei have been probed via the $(\mathrm{n}, \mathrm{p})$ reactions (W. P. Alford, private communication) and a somewhat larger number by the $(\mathrm{p}, \mathrm{n})$ reactions. For the case of transitions from a thermally excited $G T_{+}$resonance state, one can use the experimentally probed $T_{+}$distribution to appropriately weight the initial state distribution thermally. Work is in progress to utilize these data and make comparison with the $\beta^{-}$-decay or electron capture rates of FFNII and others.

\section{REFERENCES}

Bloom, S. D. \& Fuller, G. M. 1985, Nucl. Phys. A, 440, 511

Brown, B. A. \& Wildenthal, B. H. 1985, Atomic Nucl. Data Tables, 33, 347

French, J. B., Kota, V. K. B., Pandey, A. \& Tomsovic, S. 1988, Ann. Phys. (N.Y.), 181, 235

Fuller, G. M., Fowler, W. A. \& Newman, M. J. 1982, ApJS, 48, 279

Fuller, G. M., Fowler, W. A. \& Newman, M. J. 1982b, ApJ, 252, 715 (FFN II)

Gilbert, A. \& Cameron, A. G. W. 1965, Can. J. Phys, 43, 1446

Hillman, M. \& Grover, J. 1969, Phys. Rev., 185, 1303

Kar, K., Sarkar, S. \& Ray, A. 1991, Phys. Lett. B., 261, 217; 1992, Phys. Lett. B., 277, 528

Klapdor, H. V., Metzinger, J. \& Oda, T. 1990, Atomic Nucl. Data Tables, 44, 73

Kota, V. K. B. \& Kar, K. 1989, Pramana, 32, 647

Moller, P. \& Randrup, J. 1990, Nucl. Phys. A, 514, 1 
Morita, M. 1973, Beta decay and muon capture (W.A. Benjamin, Inc).

Nakayama, K., Pio Galeao, A., \& Krmpotic, F. 1982, Phys. Lett. B, 114, 217.

Nomoto, K., Shigeyama, T., Kumagai, S. \& Yamaoka, H. 1991, in "Supernovae and Stellar Evolution" eds. A. Ray \& T. Velusamy (World Scientific) 116.

Schenter, G.K. \& Vogel, P. 1983, Nucl. Sci. Engg., 83, 393.

Takahashi, K., Yamada, J. M. \& Kondoh, J. T. 1973, Atomic Nucl. Data Tables, 12, 101. von Egidy, T., Schmidt, H. \& Behkami, A. 1988, Nucl. Phys, A481, 189.

Woosley, S. E., \& Weaver, T. A. 1988, Phys. Rept., 163, 79. 